\title{
RESEARCH
}

Open Access

\section{Skeletal muscle healing by M1-like macrophages produced by transient expression of exogenous GM-CSF}

Leonardo Martins ${ }^{1}$, Camila Congentino Gallo ${ }^{1}$, Tâmisa Seeko Bandeira Honda ${ }^{1}$, Patrícia Terra Alves ${ }^{1}$, Roberta Sessa Stilhano ${ }^{2}$, Daniela Santoro Rosa ${ }^{3}$, Timothy Jon Koh ${ }^{4}$ and Sang Won $\operatorname{Han}^{1,5^{*}}$ (D)

\begin{abstract}
Background: After traumatic skeletal muscle injury, muscle healing is often incomplete and produces extensive fibrosis. The sequence of M1 and M2 macrophage accumulation and the duration of each subtype in the injured area may help to direct the relative extent of fibrogenesis and myogenesis during healing. We hypothesized that increasing the number of $\mathrm{M} 1$ macrophages early after traumatic muscle injury would produce more cellular and molecular substrates for myogenesis and fewer substrates for fibrosis, leading to better muscle healing.

Methods: To test this hypothesis, we transfected skeletal muscle with a plasmid vector to transiently express GMCSF shortly after injury to drive the polarization of macrophages towards the M1 subset. C57BL/6 mouse tibialis anterior (TA) muscles were injured by contusion and electroporated with UP-mGM, which is a plasmid vector that transiently expresses GM-CSF. Myogenesis, angiogenesis, and fibrosis were evaluated by histology,

immunohistochemistry, and RT-qPCR; subpopulations of macrophages by flow cytometry; and muscle functioning by the maximum running speed on the treadmill and the recovery of muscle mass.

Results: Muscle injury increased the number of local M1-like macrophages and decreased the number of M2-like macrophages on day 4, and UP-mGM treatment enhanced this variation. UP-mGM treatment decreased TGF- $\beta 1$ protein expression on day 4, and the Sirius Red-positive area decreased from $35.93 \pm 15.45 \%$ (no treatment) to $2.9 \% \pm 6.5 \%(p<0.01)$ on day 30. UP-mGM electroporation also increased Hgf, Hifla, and Mtor gene expression; arteriole density; and muscle fiber number during regeneration. The improvement in the quality of the muscle tissue after treatment with uP-mGM affected the increase in the TA muscle mass and the maximum running speed on a treadmill.
\end{abstract}

Conclusion: Collectively, our data show that increasing the number of M1-like macrophages immediately after traumatic muscle injury promotes muscle recovery with less fibrosis, and this can be achieved by the transient expression of GM-CSF.

Keywords: Fibrosis, Skeletal muscle, Injury, Myogenesis, GM-CSF, Macrophage, Arteriogenesis, Contusion

\footnotetext{
* Correspondence: sang.han@unifesp.br

'Interdisciplinary Center for Gene Therapy, Escola Paulista de Medicina, Universidade Federal de São Paulo, São Paulo, Brazil

${ }^{5}$ Department of Biophysics, Escola Paulista de Medicina, Universidade Federal de São Paulo, Rua Mirassol 207, São Paulo, SP 04044-010, Brazil

Full list of author information is available at the end of the article
}

(c) The Author(s). 2020 Open Access This article is licensed under a Creative Commons Attribution 4.0 International License, which permits use, sharing, adaptation, distribution and reproduction in any medium or format, as long as you give appropriate credit to the original author(s) and the source, provide a link to the Creative Commons licence, and indicate if changes were made. The images or other third party material in this article are included in the article's Creative Commons licence, unless indicated otherwise in a credit line to the material. If material is not included in the article's Creative Commons licence and your intended use is not permitted by statutory regulation or exceeds the permitted use, you will need to obtain permission directly from the copyright holder. To view a copy of this licence, visit http://creativecommons.org/licenses/by/4.0/ The Creative Commons Public Domain Dedication waiver (http://creativecommons.org/publicdomain/zero/1.0/) applies to the data made available in this article, unless otherwise stated in a credit line to the data. 


\section{Introduction}

Muscle injuries often require medical care and are difficult to treat, resulting in absences from and disabilities at work and causing a significant public health burden [1]. In sports medicine, muscle injuries are the most frequent event among athletes in both recreational and competitive activities, reaching $55 \%$ of all cases [2]. Despite advances in diagnosis and therapy, treatment of muscle injury still primarily relies on RICE (rest, ice, compression, and elevation), immobilization, and physiotherapy $[3,4]$. However, following severe muscle injury, patients suffer contracture, muscle atrophy, and reinjury because of incomplete muscle regeneration $[2,5]$. Thus, advanced therapeutic methods are needed.

The healing process in the injured skeletal muscle consists of overlapping phases of degeneration, inflammation, regeneration, and fibrosis $[2,6]$. Efficient regeneration of the injured muscle is thought to compete with fibrotic healing, and excessive fibrosis is thought to impede regeneration. This balance depends mainly on the cells and factors that are present at the degeneration and inflammation stages of healing. However, much remains to be learned about the regulation of this balance between regeneration and fibrosis.

Macrophages are the main players in the maintenance of tissue homeostasis and actively participate in tissue repair through both proinflammatory and antiinflammatory activities $[7,8]$. The apparent paradoxical activities of macrophages are due to the presence of heterogeneous subpopulations, each of which performs specific activities, and the plasticity of these cells in changing phenotypes in response to the local microenvironment and growth factor signaling $[8,9]$. Inflammatory stimuli, such as IFN- $\gamma$ and lipopolysaccharide, produce classically activated macrophages, also known as M1 macrophages, and anti-inflammatory stimuli, such as IL-4 or IL-13, produce alternatively activated macrophages, also known as M2 macrophages [8, 9].

As skeletal muscle is subjected to frequent injuries, several studies have shown the participation of macrophages in inflammatory, fibrotic, and regenerative processes $[10,11]$. Soon after muscle injury, the number of M1 macrophages rapidly increases in the injured area, and approximately 2 days later, this population is replaced by M2 macrophages [9]. In vitro studies have shown that M1 macrophages reduce collagen production via fibroblasts and stimulate myoblast proliferation $[10$, $12,13]$, while M2 macrophages increase collagen production and promote myoblast differentiation and fusion to form myofibers $[10,14]$. These facts imply that the order of M1 and M2 macrophage production and the duration of the activity of each subtype in the injured area are directly related to the extent of fibrogenesis and myogenesis, and any significant changes in these processes can result in more fibrosis and less regenerated muscle or vice versa.

Based on these observations, we hypothesized that an additional increase in the number of proinflammatory macrophages for a few days at the beginning of muscle injury should produce more myoblasts and fewer myofibroblasts, providing more substrates for myogenesis and fewer substrates for fibrosis for the emerging proregenerative macrophages. To test this hypothesis, we transfected skeletal muscle with a plasmid vector to transiently express GM-CSF (granulocyte and monocyte colony stimulating factor) soon after injury because GMCSF is known to polarize macrophages towards a proinflammatory phenotype $[15,16]$. As a consequence, we expected to observe rapid muscle recovery with less fibrosis.

\section{Methods}

\section{Research ethics committee approval}

All the animal procedures were performed in full compliance with the institutional guidelines and were approved by the Institutional Animal Care and Use Committee (number: 8866221018).

\section{Murine GM-CSF expression vector}

The murine GM-CSF (mGM-CSF) expressing plasmid vector was previously constructed in our laboratory and was named uP-mGM [17]. In this vector, mGM-CSF gene expression is promoted by the strong cytomegalovirus promoter and enhancer. For all experiments, the uP-mGM was amplified and purified using endotoxinfree plasmid purification systems (Qiagen Inc., Valencia, CA, USA).

\section{Skeletal muscle injury and in vivo gene therapy}

Muscle contusion was performed based on the method described by Kasemkijwattana et al. [6]. C57/BL6 mice aging 10-12 weeks and weighting $25 \mathrm{~g}$ were anesthetized with an IP injection of ketamine $(40 \mathrm{mg} / \mathrm{kg}$ of animal) and xylazine $(10 \mathrm{mg} / \mathrm{kg}$ of animal). The mouse was positioned in ventral decubitus and the posterior limbs positioned extending the knee and plantar flexion. A stainless-steel ball weighting $16.2 \mathrm{~g}$ with $1.6 \mathrm{~cm}$ diameter was placed on the top of a PVC tube with $100 \mathrm{~cm}$ height to fall by gravity. At the bottom of the tube, a blunttipped nail (tip area is approximately equal to the TA muscle surface) was placed so that the impact was transferred to the entire TA muscle area. During first 4 days after contusion, fentanyl $(0.05 \mathrm{mg} / \mathrm{kg}$; IP) was administered to alleviate pain. Nonsteroidal anti-inflammatory drugs (NSAIDs) were not used here because these drugs affect muscle regeneration [18].

For in vivo transfection, electroporation was performed based on our previous experience that generates 
high transfection rate $[19,20]$. Briefly, mice were anesthetized as described above and $100-\mu \mathrm{g}$ plasmids in $100 \mu \mathrm{L}$ PBS $\left(137 \mathrm{mM} \mathrm{NaCl}, 10 \mathrm{mM} \mathrm{Na} \mathrm{HPO}_{4}, 2 \mathrm{mM}\right.$ $\mathrm{KH}_{2} \mathrm{PO}_{4}, \mathrm{pH}$ 7.3) were injected intramuscularly using an insulin syringe. A pair of needle electrodes was introduced flanking the plasmid injected area, and 3 pulses of $80 \mathrm{~V}$ with $20 \mathrm{~ms}$ duration and $1 \mathrm{~s}$ intervals between pulses were applied (Eletroporator T820-BTX Genetronics, San Diego, USA). After electroporation, the mice were kept in the microisolators with ad libitum access to food and water.

\section{Histology}

Mice were euthanized by cervical dislocation, and tibialis anterior (TA) muscles were collected for histology following previously established protocols [21]. Paraffinembedded tissues were sectioned $(4.5 \mu \mathrm{m})$ and stained with hematoxylin-eosin and Sirius Red. Silanized glass slides were used for immunohistochemistry (IHC) using the antibodies shown in Additional file 1. For histomorphometry, all images were captured inside injured areas and 20 randomly selected $200 \times$ or $400 \times$ high-powered fields from each sample. The percentages of cells expressing each marker of S1 were obtained after image acquisition with an optical microscope (Olympus BX60, Center Valley, PA, USA) and quantified using Image-Pro Plus $^{\circ}$ (Media Cybernetics, Rockville, MD, USA) according to a previously described protocol [21]. Predetermined histomorphological criteria (see Additional file 2) were used for histomorphology.

\section{Real-time RT-PCR for quantitative gene expression analysis}

Mice were euthanized by cervical dislocation to collect TA muscles. Total RNA from TA muscles was extracted using RNeasy ${ }^{\circ}$ Microarray Tissue, and the cDNA was synthesized using $\mathrm{RT}^{2}$ First Strand Kit (Qiagen). For RTqPCR, we used PCR array provided by the Qiagen (see Additional file 3). GAPDH gene was used as internal control. Each biological sample had a single replicate reaction, and two biological samples were used to all of them. Relative gene expression was calculated by $2^{-\Delta \Delta C T}$. The changes in mRNA expression were expressed as fold changes relative to control.

\section{Flow cytometry}

The TA muscles of the right and left limbs were collected on days $0,4,7$, and 15 after injury, combined or not with electroporation with uP-mGM $(100 \mu \mathrm{g} / 100 \mu \mathrm{L}$ PBS), as described above. Muscles were incubated with $0.2 \%$ collagenase $1 \mathrm{~A}$ solution (Sigma Aldrich) at $30^{\circ} \mathrm{C} /$ $45 \mathrm{~min}$. Collagenase activity was blocked by the addition of $5 \mathrm{~mL}$ DMEM:FBS (1:1). The samples were filtered in cell strainer 70 and $40 \mu \mathrm{m}$.
Isolated cells were washed and resuspended with $0.5 \%$ PBS-BSA and preincubated with anti-CD16/32 antibody (eBioscience). Cells were incubated at $4{ }^{\circ} \mathrm{C} / 30 \mathrm{~min}$ with the following antibodies: eFluor 450 anti-CD45 (clone 30-F11, eBioscience, catalog number 48-0451-82), FITC anti-F4/80 (clone BM8, eBioscience, catalog number 114801-82), APC-eFluor 780 anti-MHCII (clone M5/ 114.15.2, eBioscience, catalog number 47-5321-82), and PE anti-CD206 (clone C068C2, BioLegend, catalog number 141706). Dead cells were excluded by Fixable Viability Dye efluor 506 (eBioscience) labeling and fixed in 1\% buffered PFA for $15 \mathrm{~min}$. Flow cytometry was performed using FACSCanto II (4.2.2) (BD Bioscience), and data were analyzed using FlowJo software (version 9.2). In this study, CD45+F4/80+MHCII+CD206- macrophages were considered as M1-like [22-24] and CD45+F4/80+ MHCII-CD206+ as M2-like [23-25].

\section{Muscle function testing}

Running capacity was tested in all groups. All animals underwent a 5-day motorized treadmill adaptation period (Exer 3/6 Treadmill, Columbus Instruments, Ohio, USA) prior to random separation between groups. Once a day, they ran for $15 \mathrm{~min}$ at a speed of $10 \mathrm{~m} / \mathrm{min}$. In addition to allowing the adaptation of the animals of the exercised groups to the training and handling conditions, this period also allowed to select the non-runner running animals. After this time, we performed the forced exercise capacity, which allowed to infer the physical performance of the animals by determining the maximum speed reached. The test was performed $1,4,7,15$, and 30 days after the last session of adaptation. Mice were then tested to exhaustion, defined as the point when mice would no longer run for more than $2 \mathrm{~s}$ at a time preferring to sit on the shock grid even when touched by a gloved hand. The steps of the test protocol were as follows: mice were started running at $5 \mathrm{~m} / \mathrm{min}$ for the first $3 \mathrm{~min}$, which increased to $10 \mathrm{~m} / \mathrm{min}$ for 1 min, then increased by $1 \mathrm{~m} / \mathrm{min}$ increments every 3 min thereafter until exhaustion [26, 27]. After this, all animals had a rest period of $24 \mathrm{~h}$ before any experimental procedure. After euthanasia of the animals, the tibialis anterior (TA) muscle was excised and its mass measured in an analytical balance.

\section{Statistical analysis}

All the statistical analyses were performed in GraphPad Prism (Version 6.0 GraphPad Software Inc., San Diego, USA). The results are expressed as the mean \pm SD. Differences were considered statistically significant at $p<0.05$. The statistical methods used are detailed in the figure legends. 


\section{Results}

\section{Effects of injury and transfection on the expression of GM-CSF and its receptors}

Mouse TA muscles were subjected to traumatic injury and transfected with $\mathrm{uP}-\mathrm{mGM}$ vectors via electroporation, as shown in the schematic diagram in Fig. 1. The injured area was red and swollen soon after impact, but no sign of bone fracture was observed during the manual examination. The swelling lasted approximately 4 days, and the reddened area completely disappeared on the 7th day.

The expression of Gm-csf and its receptors was analyzed by RT-qPCR. Healthy TA muscles expressed very low levels of Gm-csf and its receptors (Gm-csfra and Gm-csfrb). As expected, the expression of Gm-csf was dramatically increased $24 \mathrm{~h}$ after electroporation (GM) (279.41 \pm 139.34 -fold). Interestingly, the expression of Gm-csfra and Gm-csfrb was also increased (123.21 \pm 1.28 - and $270.77 \pm 55.50$-fold, respectively). The expression of these genes returned to basal levels after 1 week (Fig. 2A). When TA muscles were subjected to both injury and uP-mGM electroporation (LM+GM), the Gmcsf expression profile was similar to that of the GM group, but it lasted longer. The Gm-csf receptors in the $\mathrm{LM}+\mathrm{GM}$ group were expressed at higher levels than those in the GM group and followed a similar decay. Injury itself also induced the expression of the Gm-csf receptors, but the levels were well below those induced by transfection with uP-mGM.

\section{Effect of uP-mGM transfection on muscle degeneration,} inflammation, and regeneration

Muscle healing after injury was evaluated by quantifying the degenerated and regenerated muscle fibers, the injured muscle area, and the number of infiltrated leukocytes in HE-stained muscle sections, following previously defined criteria [21] (Fig. 2B, C and Additional file 2). Following injury alone (LM), progressive increases in the number of infiltrated leukocytes and the injured area were observed up to day 7 , followed by rapid decreases (Fig. 2C). Electroporation of uP-mGM into non-injured muscle (GM) also resulted in some leukocyte infiltration and muscle damage but too much lesser extents than those observed in traumatic injury. Importantly, uPmGM electroporation into traumatically injured muscle $(\mathrm{LM}+\mathrm{GM})$ led to a significant reduction in the injured area starting on day 4 , and the injured area reached basal levels on day 15. uP-mGM electroporation also reduced the number of infiltrated leukocytes compared to traumatic injury alone.

Within the lesion area, the LM group showed a predominance of degenerated muscle fibers after day 7, while the LM+GM group showed very low levels of degenerated fibers and high levels of normal and regenerating muscle fibers. Within the smaller lesions of the muscles transfected with GM-CSF alone (GM), normal and regenerated fibers were abundant. Healthy muscles electroporated with uP vector did not significantly alter the quality and quantity of the muscle fiber (see Additional file 4). Thus, GM-CSF transfection appears to promote a regenerative healing response.

Effect of uP-mGM transfection on muscle fibrosis analysis To analyze fibrosis, we first performed Sirius Red staining to evaluate the total collagen content. In healthy skeletal muscle, the Sirius Red-stained area was minimal, but after injury, the stained area progressively increased,
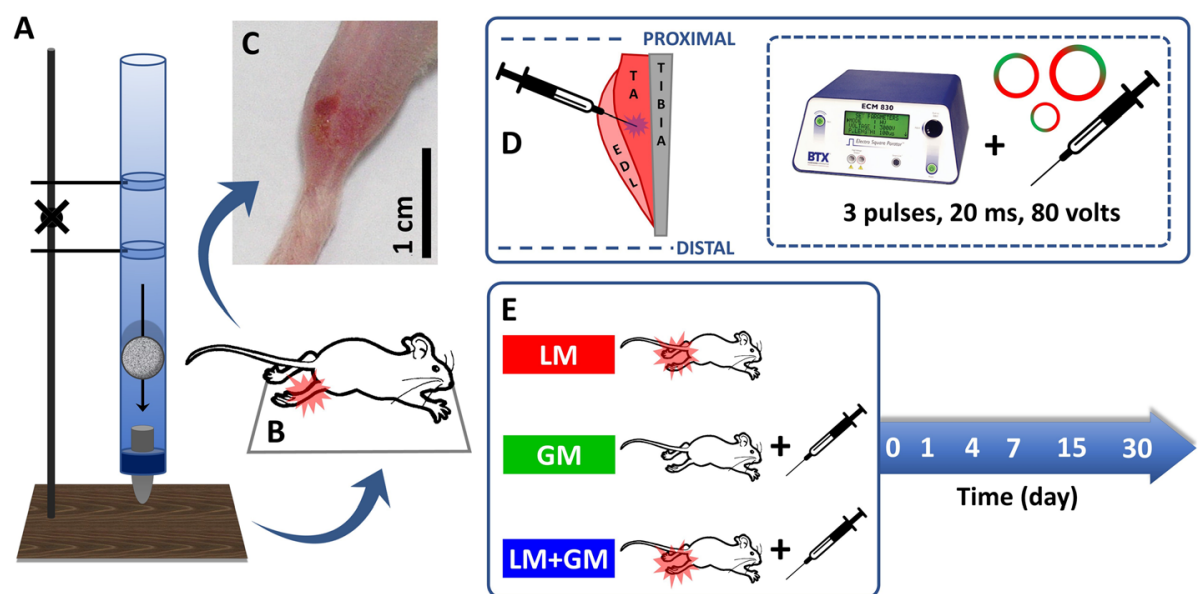

Fig. 1 Illustration of skeletal muscle injury and gene therapy. (a) The height of the device was adjusted to $100 \mathrm{~cm}$ to transfer the $16.2-\mathrm{g}$ projectile to the (b) tibialis anterior (TA) muscle of the animal placed in the supine position. (c) Representative image of an injured leg after $24 \mathrm{~h}$ and (d) representative scheme of the injection of the plasmid vectors into the central portion of the TA muscle; the overlapping structures, extensor digitorum longus (EDL), and tibial bone are also shown. (e) Experimental timeline and animal groups: GM, healthy mouse treated with GM-CSF; $L M$, injured mouse without treatment; $L M+G M$, injured mouse treated with GM-CSF 


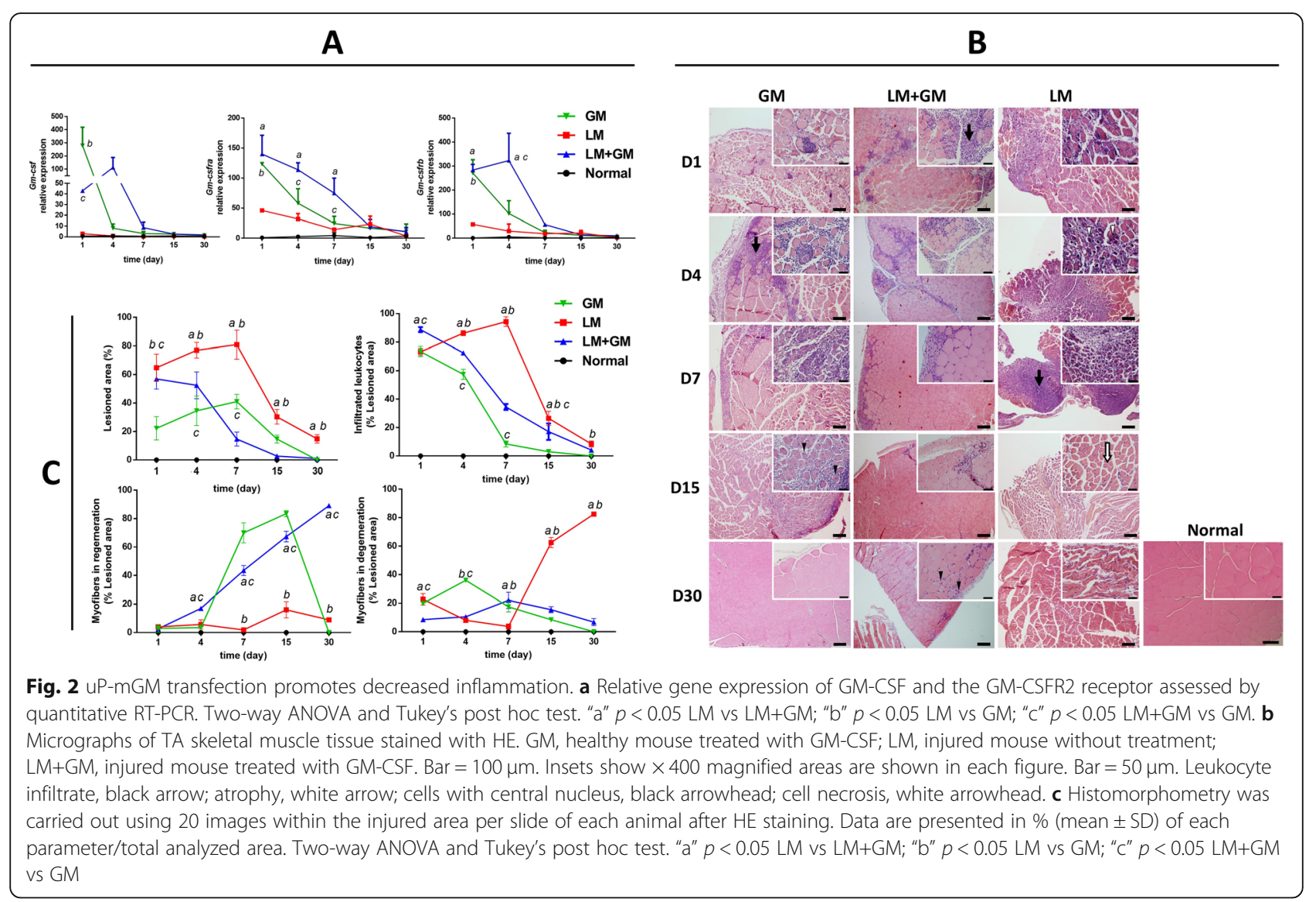

reaching $35.93 \pm 15.45 \%$ of the muscle area on day 30 (Fig. 3A). Thus, the contusion injury model results in significant skeletal muscle fibrosis. Importantly, GMCSF treatment (LM+GM) significantly inhibited fibrosis, as the Sirius Red-stained area was maintained close to that observed at baseline. GM-CSF overexpression in healthy muscle did not significantly affect fibrosis.

To better understand the mechanisms involved in fibrosis after traumatic injury, muscle sections were stained with anti-TGF- $\beta 1$ and TGF- $\beta 3$ antibodies (Fig. 3B). In the LM mice, the TGF- $\beta 1$-stained area increased quickly after injury and then decreased over time. GM-CSF treatment drastically reduced the TGF$\beta 1$-stained area. However, the TGF- $\beta 3$-stained area showed an inverse response to GM-CSF compared to the TGF- $\beta 1$-stained area (Fig. 3B). LM muscles exhibited a peak expression on day 1 , which subsequently disappeared, but the GM-CSF-treated $(\mathrm{LM}+\mathrm{GM})$ muscles showed significantly enhanced TGF- $\beta 3$ levels on days 4 and 7 postinjury. The expression of GM-CSF in healthy muscle (the GM group) generated TGF- $\beta 1$ and 3 profiles that were similar to those generated by the expression of GM-CSF in injured muscle (the $\mathrm{LM}+\mathrm{GM}$ group). In summary, the expression profile of TGF- $\beta 1$ is compatible with the promotion of fibrosis observed by Sirius
Red staining, whereas the expression profile of TGF- $\beta 3$ is associated with antifibrosis.

The expression of fibrosis-related genes was also assessed by RT-qPCR. The expression of $T g f b 1$ rapidly increased after muscle injury (LM) and returned to baseline by day 30 . Transfection of uP-mGM into healthy muscle also caused a rapid increase in the expression of $T g f b 1$. Transfection into the injured muscle $(\mathrm{LM}+\mathrm{GM})$ resulted in an expression profile over time that was similar to that induced by transfection into healthy muscle, but the expression levels tended to be amplified. On the other hand, the expression of the TGF- $\beta 1$ receptors Tgfbr 1 and Tgfbr2 was high in all the groups only during the first 7 days, and only the LM group maintained high gene expression levels thereafter, while the other groups maintained baseline gene expression levels (Fig. 3C). Therefore, the expression profiles of TGF- $\beta 1$ mRNA and protein were not similar.

Col1a1, which is a marker of fibrosis, was overexpressed in traumatically injured muscle, reaching a peak at day 7 and slowly returning to baseline at day 30 . The expression of Col1a1 was significantly reduced in the LM+GM group on days 7 and 15 postinjury (Fig. 3C). In contrast, the Col3a1 gene expression profile was similar 


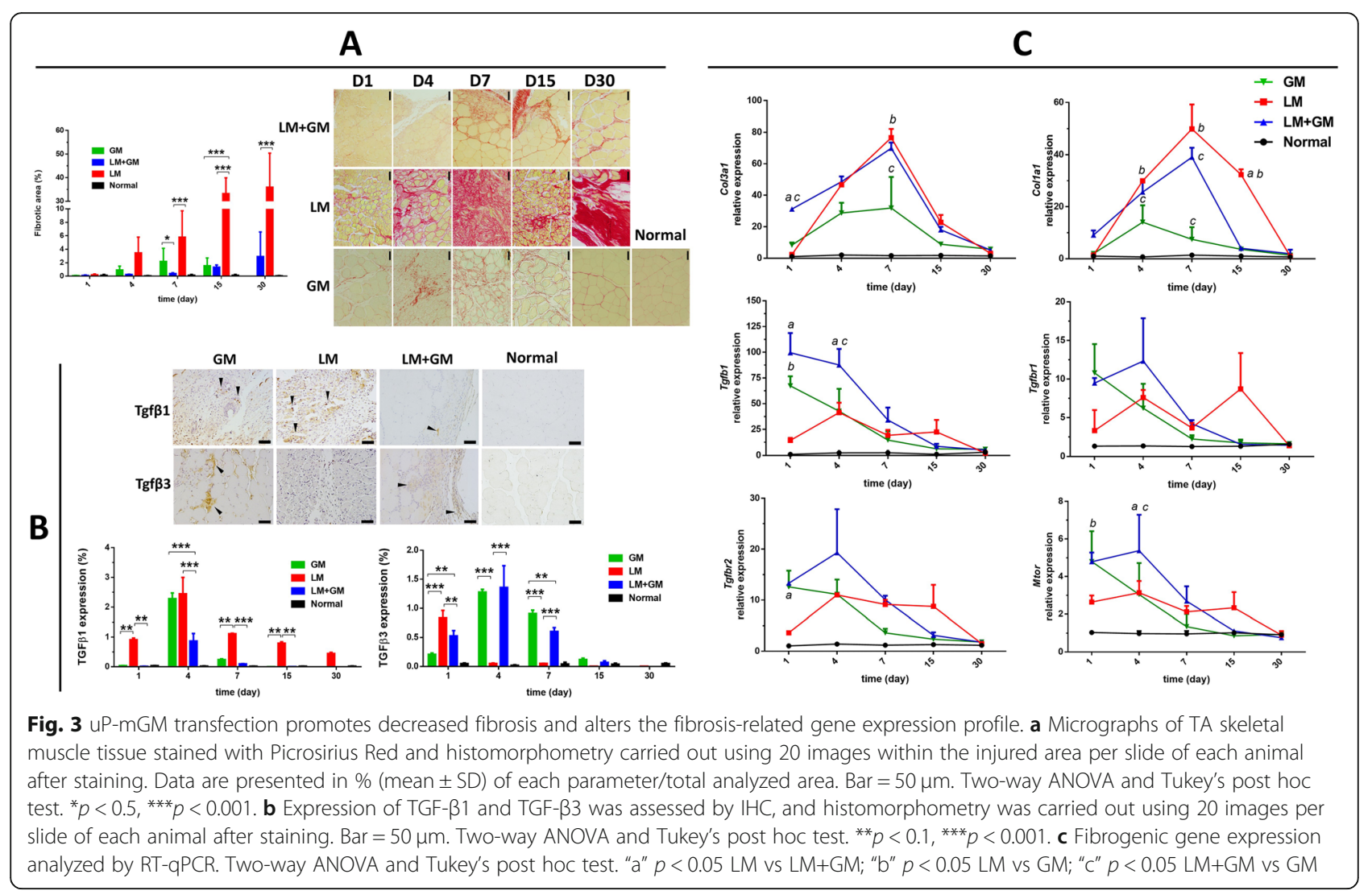

between the LM and LM+GM groups, and the expression in both groups peaked on day 7 and returned to baseline on day 30 .

mTOR is a key regulator in the maintenance of skeletal muscle and regeneration after injury. Muscle injury elevated Mtor expression, but transfection with uPmGM promoted much higher Mtor expression, which returned to baseline levels after 1 week.

\section{Effect of uP-mGM transfection on angiogenesis}

Angiogenesis was evaluated in muscle sections by identifying vessel-like structures using an anti- $\alpha$-SMA antibody (arterioles) and Isolectin IB4 staining (capillaries). Traumatic injury alone (LM) resulted in very few $\alpha$ SMA-positive vessels at all time points, whereas uPmGM transfection resulted in increased a-SMA-positive vessels beginning on day 4 , which persisted until day 30 in both the LM and LM+GM groups (Fig. 4A).

Measurements of capillary density showed similar trends among the LM, LM+GM, and GM groups, reaching a peak on day 4 and decreasing to baseline on day 30. Among all the time points investigated, uP-mGM transfection only increased the capillary density on day 4 postinjury. Therefore, uP-mGM transfection appears to have primarily promoted arteriogenesis and to have a smaller effect on angiogenesis.
The expression of angiogenic genes was assessed by RT-qPCR using muscle samples from each time point. Among the 6 genes (Hifla, Hgf, Kdr, Flt1, Vegfa, and Fgf1) assessed, traumatic injury (in the LM and LM+GM groups) induced a significant increase in the expression of Hifla and $H g f$, which reached a maximum value on day 4. Importantly, transfection with uP-mGM produced a further significant increase in these genes.

In addition, transfection with uP-mGM increased the expression of the Vegfa receptor $K d r$ on day 1 after electroporation in both the healthy and LM groups, but this expression returned to baseline on day 4 . Therefore, the increase in $\alpha$ SMA-positive vessel density could be related to the increase in $\mathrm{HIF} \alpha$, which is the main regulator of angiogenesis [28], and HGF, which promotes arteriogenesis and angiogenesis [29].

\section{Effect of uP-mGM transfection on macrophage subpopulations}

The macrophage subpopulations in the muscles were assessed by flow cytometry. First, we gated out debris in the SSC (side scatter) vs FSC (forward scatter) plots; then gated on singlets, live cells, and CD45+ and F4/80+ cells; and finally identified subpopulations using MHCII and CD206 as markers for M1-like and M2-like macrophages, respectively (Fig. 5A). A typical dot plot showed 4 distinct populations based on the expression of MHCII and CD206: 


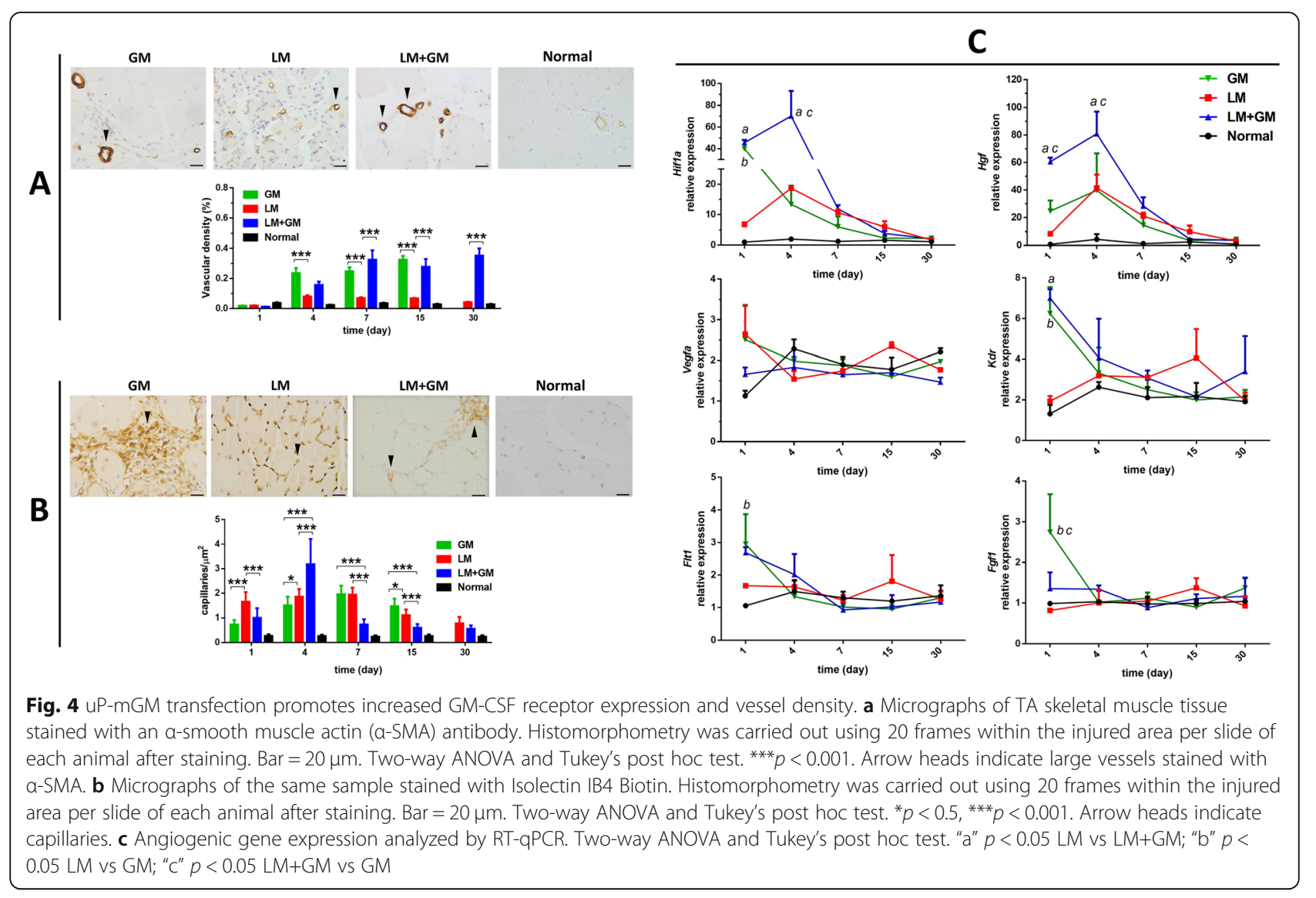

$\mathrm{MHCII}^{+} \mathrm{CD}^{206}{ }^{-}$(M1-like), $\mathrm{MHCII}^{-} \mathrm{CD}^{206}{ }^{+}$(M2-like), $\mathrm{MHCII}^{+} \mathrm{CD}_{206}{ }^{+}$(hybrid), and $\mathrm{MHC}^{-} \mathrm{CD}_{206}^{-}$(non-expressor). In non-injured muscle, most macrophages were $\mathrm{MHCII}^{+} \mathrm{CD}_{206}{ }^{+}$, with lower frequencies of $\mathrm{MHCII}^{+} \mathrm{CD} 206^{-}$ and $\mathrm{MHCII}^{-} \mathrm{CD}^{-06^{-}}$cells and a barely detectable number of $\mathrm{MHCII}^{-} \mathrm{CD}^{206^{+}}$macrophages (Fig. 5B, C). Muscle injury caused a rapid increase in the frequency of $\mathrm{MHCII}^{-} \mathrm{CD} 206^{+}$ macrophages that peaked on day 4 and slowly decreased over time, reaching baseline on day $15 . \mathrm{MHCII}^{+} \mathrm{CD} 206^{-}$ macrophages (Fig. 5B) behaved differently, slowly increasing after injury throughout the time points examined (Fig. 5C). In contrast, the $\mathrm{MHCII}^{+} \mathrm{CD}_{20} 6^{+}$macrophage population rapidly decreased on day 4 and recovered over time. Thus, $\mathrm{MHCII}^{+} \mathrm{CD}_{206^{+}}$macrophages may serve as a buffer, providing $\mathrm{MHCII}^{+} \mathrm{CD}^{2} 26^{-}$and $\mathrm{MHCII}^{-} \mathrm{CD} 206^{+}$cells, as these are required during the repair process. This speculation should be tested in future studies.

As expected, transfection with uP-mGM caused a rapid increase in the number of $\mathrm{MHCII}^{+} \mathrm{CD} 206^{-}$macrophages, which peaked on day 4 postinjury. GM-CSF overexpression in injured muscle amplified the increased numbers of this macrophage subset (day 4), which then decreased to baseline on day 7 and increased again on day 15 to a level similar to that observed in the LM group. Thus, it appears that the first increase in the numbers of $\mathrm{MHCII}^{+} \mathrm{CD} 206^{-}$macrophages is due to GM-CSF overexpression, and the second increase is due to injury.

\section{Muscle mass and functional recovery}

As muscle injury affects muscle mass and function, a treadmill running performance test was used to assess muscle function, after which the TA muscles were harvested and weighed.

Traumatic injury resulted in a progressive loss of muscle mass soon after injury, remarkably peaked on day 7, followed by gradual recovery (Fig. 6). Importantly, muscle mass did not recover to baseline levels by day 30 postinjury. GM-CSF-treated mice showed a similar loss in mass but a more complete recovery, reaching baseline values by day 30 .

With regard to muscle function, the maximum treadmill running speed dramatically decreased after injury and returned to baseline by day 30 postinjury (Fig. 6).

The GM-CSF-treated group followed a profile similar to that of the LM group, but the recovery speed was faster. However, the variations in muscle mass and speed recovery did not exhibit statistical significance within groups at different adjacent time points or between the LM and LM+GM groups at each time point. 

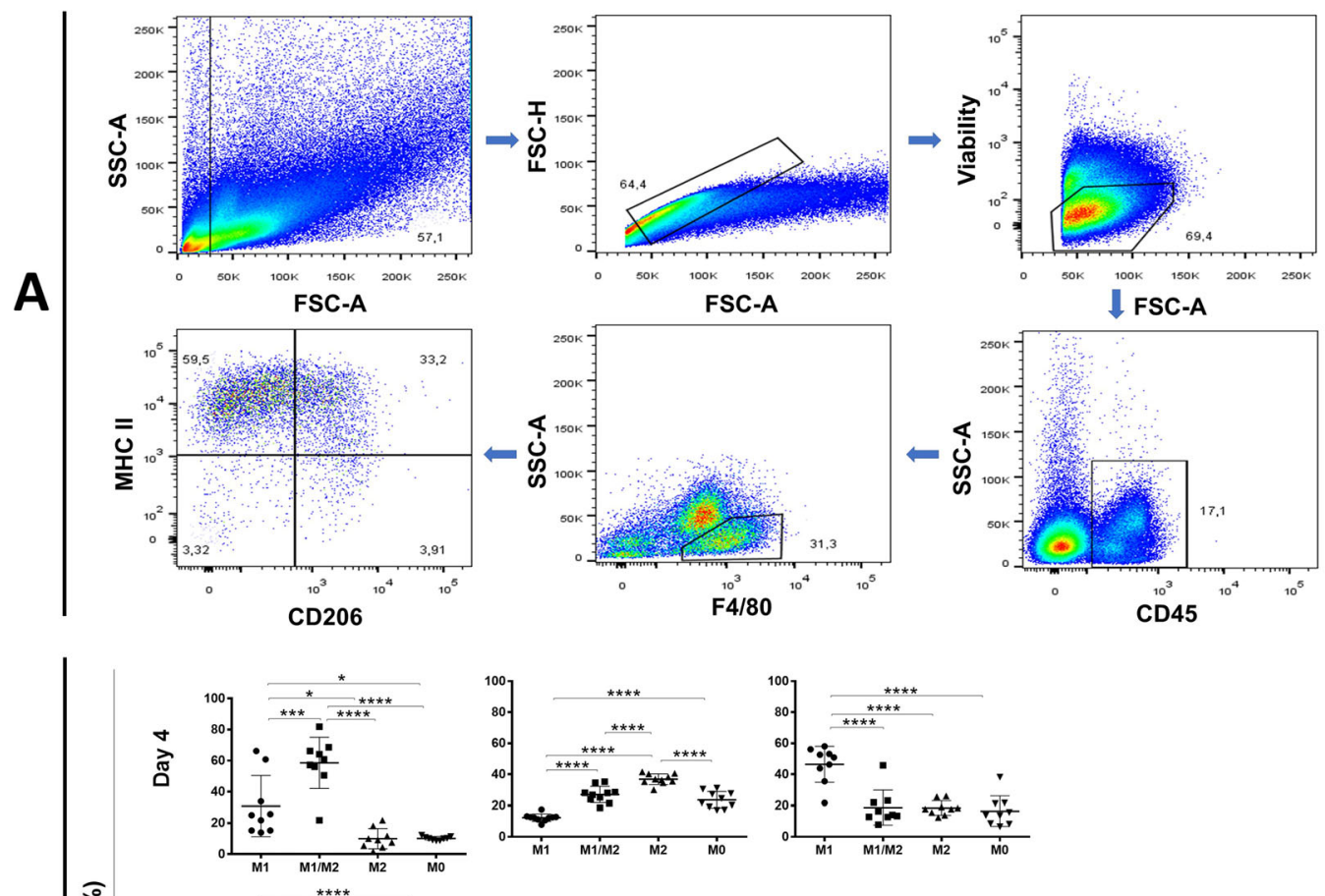

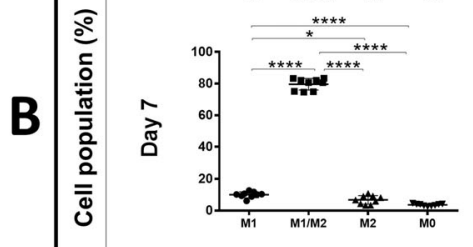
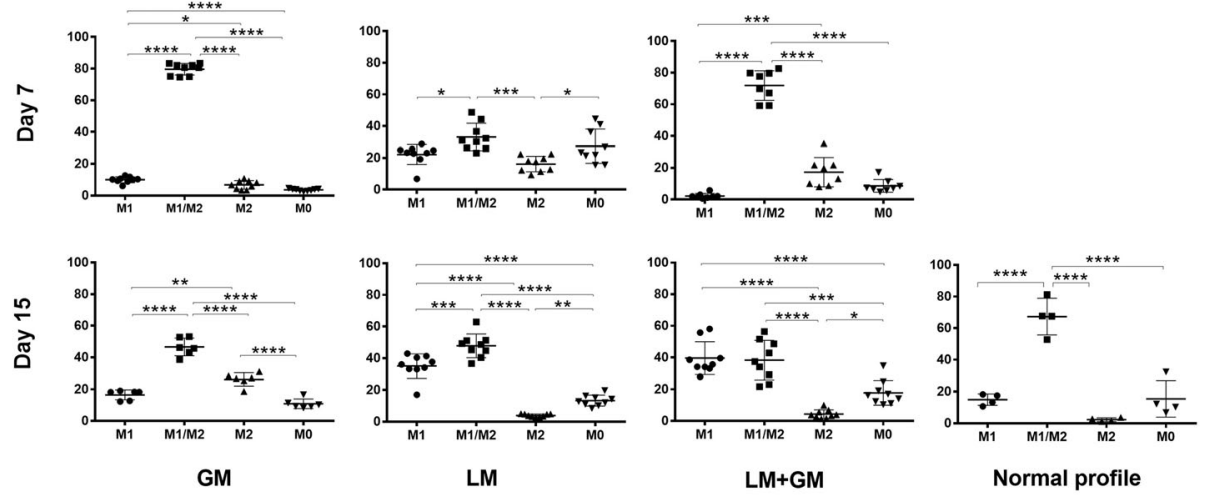

Treatment groups
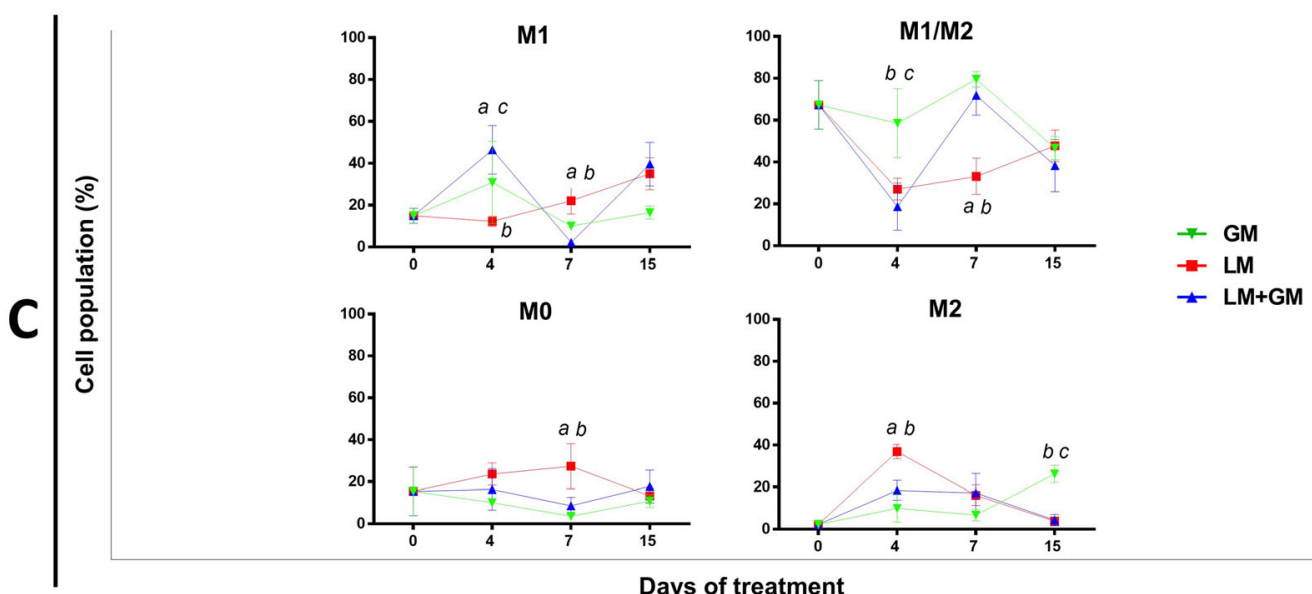

Days of treatment

Fig. 5 (See legend on next page.) 
(See figure on previous page.)

Fig. 5 uP-mGM transfection leads to increased numbers of the M2 macrophage subtype. a Flow cytometry analysis of the macrophage subpopulations. Gating strategy used to identify M0 (CD45 $\left.{ }^{+} \mathrm{F} 4 / 80^{+} \mathrm{CD} 206^{-} \mathrm{MHCl}-\mathrm{I}^{-}\right), \mathrm{M} 1\left(\mathrm{CD} 45^{+} \mathrm{F} 4 / 80^{+} \mathrm{CD}_{206^{-}} \mathrm{MHCII}^{+}\right), \mathrm{M}^{2}\left(\mathrm{CD} 45^{+} \mathrm{F} 4 / 80^{+}\right.$

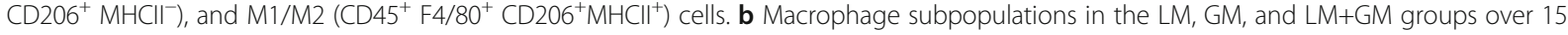
days. The normal group represents mice that did not undergo injury and treatment. c Variation in the numbers of macrophages per subpopulation over time. Time zero is represented by macrophages from the normal group, as mentioned above (b). Two-way ANOVA and Tukey's post hoc test. "a" $p<0.05$ LM vs LM+GM; "b" $p<0.05$ LM vs GM; "c" $p<0.05$ LM+GM vs GM

\section{Discussion}

Full restoration of skeletal muscle structure and function after traumatic injury requires several different cell types and numerous molecules working together to efficiently control the damaged tissue through each phase of healing. Macrophages are known to play essential roles in each phase of efficient muscle regeneration, but the regulation of macrophage subsets may be dysregulated in traumatic injury, resulting in extensive fibrosis [10, 11].

GM-CSF polarizes macrophages towards a proinflammatory phenotype (see Additional file 5) $[15,16]$, and in our study, this phenomenon can be observed by the increase in M1-like macrophages and decrease in M2-like macrophages on day 4 (Fig. 5B, C). The variations in this cell population over 15 days were very similar in the GM and $\mathrm{LM}+\mathrm{GM}$ groups but were enhanced in the $\mathrm{LM}+\mathrm{GM}$ group. These results show that the balance of the M1like and M2-like populations can be disrupted by GMCSF, favoring the formation of more M1-like cells. It is also important to note that this disruption lasted only 1 week and peaked at day 4 after electroporation, which is in accordance with the GM-CSF expression profile (Fig. 2A). The non-integrative plasmid vector-mediated gene expression in skeletal muscle usually lasts approximately 1 week, and after this time, a very low level of expression is maintained [30]. Therefore, the temporal increase in the number of M1-like macrophages as a consequence of electroporation at the beginning of muscle injury achieves our initial goals, namely, more myogenic activity and less fibrogenic activity.

Here, we used the terms M1-like and M2-like, which indicate proinflammatory and anti-inflammatory macrophages, respectively, although we acknowledge that these subtypes are only points on a continuum of macrophage phenotypes. Most cell markers have been identified using cell cultures, and cell media are well defined; however, in vivo macrophage phenotypes are likely more complex because they are exposed to a complex and rapidly changing microenvironment following injury. For example, Novak et al. [11] reported that in traumatically injured muscle, macrophages do not exhibit the canonical M1 or M2 phenotypes, and M1- and M2a-associated genes are coincidentally upregulated soon after injury, leading to difficulty in classifying macrophages and variation in the results from different authors.

We chose GM-CSF to prove our hypothesis because in addition to stimulating the proliferation and differentiation of monocytes/macrophages and polarizing macrophages towards a proinflammatory phenotype $[15,16]$, GM-CSF also mobilizes endothelial progenitor cells for vasculogenesis [31] and stimulates the proliferation of

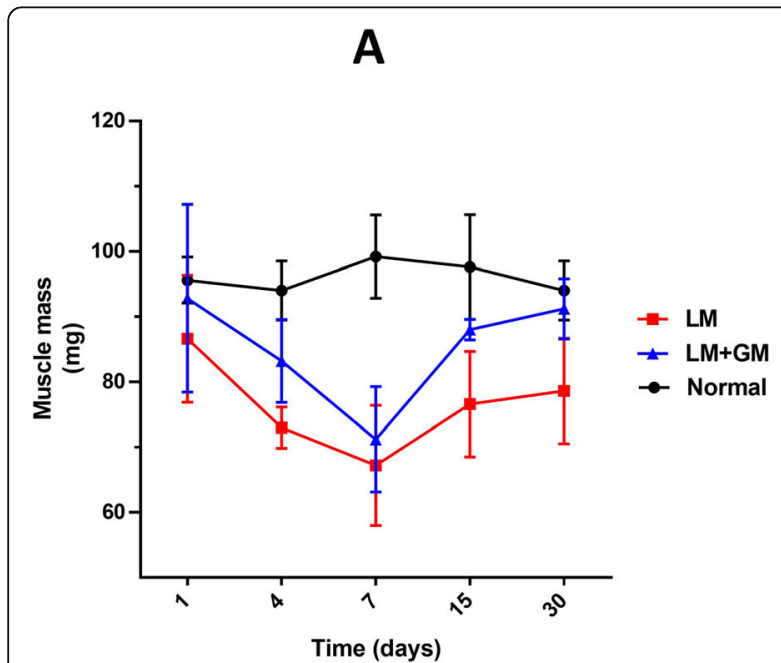

B

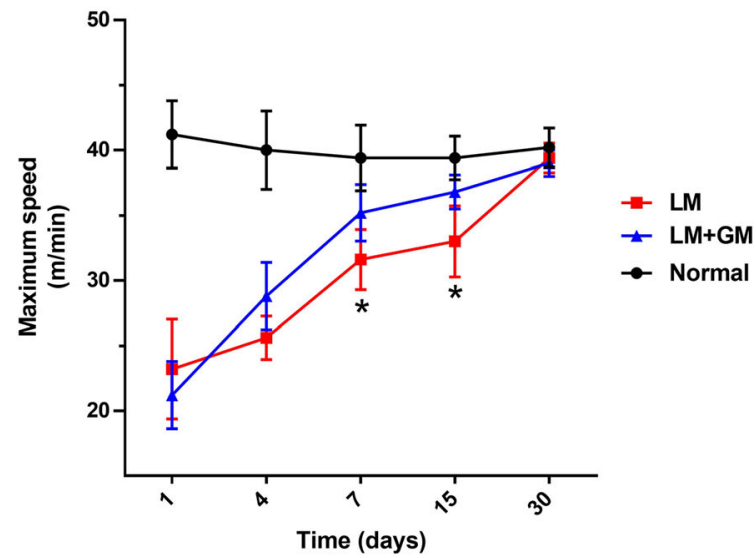

Fig. 6 uP-mGM transfection promotes recovery of muscle functional. a TA muscle mass was determined 30 days after transfection. $\mathbf{b}$ Performance of incremental maximal exercise was assessed in mice forced to run to maximum exhaustion using a standardized protocol of incremental speeds and $0^{\circ}$ inclination of the treadmill. Two-way ANOVA and Tukey's post hoc test. ${ }^{*} p<0.05 \mathrm{LM}$ vs LM+GM. Data presented as mean \pm SD 
neutrophils that express various angiogenic growth factors, such as HGF [32]. The increase in HGF expression (Fig. 4C) and vessel density (Fig. 4A) shows the roles of GM-CSF activities in the formation and remodeling of vessels to become larger and more functional, as has also been seen by others [33, 34]. HGF also triggers activation of satellite cells [35]; consequently, it participates in myogenesis. As GM-CSF regulates the development of dendritic cells, this factor has shown promise for cancer therapies [36, 37]. Thus, people who are concerned about the use of growth factor genes for angiogenic gene therapy, because these factors can stimulate tumorigenesis, are much less concerned about GM-CSF-based therapy. Therefore, a collection of information from different sources shows that GM-CSF can be a crucial factor to resolve injured skeletal muscle if appropriately administered.

The biological effects of GM-CSF can occur only if there is a sufficient concentration of GM-CSF and its receptor on the cell membrane. The GM-CSF receptor is composed of two subunits: subunit $\alpha$, which binds with high affinity to GM-CSF, and subunit $\beta \mathrm{c}$, which is responsible for signal transduction [38, 39]. This receptor is mostly expressed in myeloid cells, such as neutrophils, monocytes, and macrophages [38], and in dendritic cells [40]. When GM-CSF binds to its receptor, this complex is internalized to be recycled and partially degraded, and this process seems to stimulate synthesis of its receptors [41]. This observation can explain, at least in part, the temporal increase in the mRNA concentration of the GM-CSF receptor subunits in the muscle after electroporation (Fig. 2A).

It was shown that GM-CSF concentrations lower than $10 \mathrm{pM}$ promote hematopoietic cell survival (extended lifetime) by the phosphorylation of the subunit $\beta_{\mathrm{cSe}}{ }^{585}$. However, if this concentration is increased above $10 \mathrm{pM}$, then this Ser is dephosphorylated, and $\beta c \mathrm{Tyr}^{577}$ is phosphorylated, which increases cell survival and proliferation [42]. In our previous study, we observed $8.6 \pm 3.8 \mathrm{ng}$ of GM-CSF in a thigh muscle weighing $160 \pm 23 \mathrm{mg}$ after electroporation with uP-mGM [17]. Considering the physiological conditions and the molecular properties of GM-CSF, we observed a concentration of approximately $2.6 \mu \mathrm{M}$ (see Additional file 6). This concentration is well above $10 \mathrm{pM}$; therefore, electroporation with uP-mGM must produce enough GM-CSF to stimulate the proliferation, survival, and functional activities of hematopoietic cells. A direct proof of this assumption is the increase in the number of macrophages on day 4 after electroporation into the healthy (GM) and injured-muscle (LM+ GM) mice (Fig. 5B, C).

Resident macrophages are rare in healthy muscles and are located mainly in the epimysium and perimysium [43-46]. These resident macrophages likely have essential roles in the recruitment of circulating monocytes because the ablation of resident macrophages reduces monocyte recruitment to toxin-injured muscle [43]. As GM-CSF stimulates the proliferation of macrophages irrespective of origin, any increase in the number of local macrophages induced by GM-CSF overexpression likely includes a combination of resident and monocyte-derived macrophages. As our goal was to evaluate the effect of GM-CSF overexpression on macrophages present in the injured muscle regardless of origin, we used F4/80 as an initial marker and then used CD206 and MHCII as markers of M2-like and M1-like macrophages, respectively $[24,47,48]$.

One of the main consequences of tissue injury is fibrosis, which is a common biological response to the rapid repair of damaged tissue. However, fibrosis affects tissue function, and in some cases, it can severely impair function and even lead to death, such as in the heart and kidney $[49,50]$. As shown in Fig. 3, traumatically injured muscle developed exuberant fibrosis, which was almost completely reduced by GM-CSF overexpression. TGF- $\beta 1$ is a main player in fibrosis [51-53]. TGF- $\beta 1$ also participates in myoblast proliferation [54], but it also delays myoblast differentiation and fusion to make new myofibers [55]. Therefore, it seems that higher expression of TGF- $\beta 1$ at the beginning of muscle injury and lower expression later promote more myogenesis and less fibrogenesis; this expression profile can be observed after GM-CSF treatment (Fig. 3C). The increase in TGF- $\beta 1$ expression observed soon after uP-mGM transfection is probably due to stimulation by GM-CSF and autoregulation of TGF- $\beta 1[56,57]$. The correlation between these two factors can be observed by the similarity of their RNA gene expression patterns (Figs. 2A and 3C). However, the TGF- $\beta 1$ protein expression pattern observed by IHC is significantly different from the RNA gene expression pattern. Most likely, the antibody we used for IHC does not recognize one of the TGF- $\beta 1$ forms (active and latent complex) well [58]. In addition, other factors, such as the different stabilities of the mRNA and protein of the same factor and the rate of translation, can cause variation in concentrations $[59,60]$. Importantly, both data support the correlation between GM-CSF and TGF- $\beta 1$ and fibrosis. The reduction in fibrosis can also be due to the elevation in expression of TGF- $\beta 3$ (Fig. 3B), which belongs to the same TGF- $\beta$ family but can reduce fibrogenesis [61]. The antifibrotic activity of TGF- $\beta 3$ has also been used to treat skin scarring $[62,63]$.

Muscle mass homeostasis depends on metabolic rates, and several studies have shown that mTOR positively regulates muscle protein synthesis and, consequently, muscle hypertrophy [64]. In humans, mTORC1 activation induces myofibrillar muscle protein synthesis and muscle hypertrophy after physical exercise or ingestion 
of essential amino acids, such as leucine [65]. The involvement of mTOR was also shown in the regenerative process of severely injured muscle [66], which can also be observed in our injury model (Fig. 2C). Overexpression of GM-CSF enhanced the increase in mTOR expression, especially during the first week after electroporation, which should have significantly contributed to the increase in regenerating muscle fibers and the decrease in degenerating muscle fibers (Fig. 2C). The consequence of the increased mTOR expression and accelerated muscle recovery over time after GM-CSF administration was also reflected in the tendency to increase muscle mass (Fig. 6a) and the faster recovery of muscle function (Fig. 6b).

\section{Conclusion}

Our data show that the temporal increase in the number of M1-like macrophages soon after traumatic muscle injury is important for the recovery of skeletal muscle with less fibrosis, and this can be achieved by the transient expression of GM-CSF.

\section{Supplementary Information}

Supplementary information accompanies this paper at https://doi.org/10. 1186/s13287-020-01992-1.

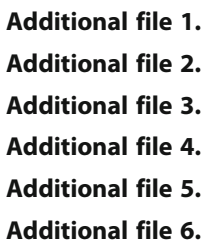

\section{Abbreviations}

GM-CSF: Granulocyte macrophage-colony stimulating factor; TA: Tibialis anterior; HE: Hematoxylin-eosin staining; IHC: Immunohistochemistry; PBS: Phosphate-buffered saline; RT-PCR: Reverse transcriptase-polymerase chain reaction; TGF- $\beta 1$ : Transforming growth factor beta 1; TGF- $\beta 3$ : Transforming growth factor beta 3; a-SMA: a-smooth muscle actin; UP-mGM: UP vector expressing murine GM-CSF

\section{Acknowledgements}

None

\section{Authors' contributions}

The authors' contributions were as follows: study design-LM and $\mathrm{SWH}$; data collection-LM, CCG, TSBH, PTA, RSS, and DSR; data analysis and interpretation-LM, CCG, TSBH, PTA, RSS, DSR, TJK, and SWH; generation of figures-LM, CCG, TSBH, PTA, RSS, DSR, and SWH; and writing of the manuscript-LM, TJK, and SWH. The authors read and approved the final manuscript.

\section{Funding}

LM, TSBH, and PTA were recipients of grants from FAPESP (11/15417-9, 16/ 04229-0, and 18/09203-5). This work was supported by FAPESP (2015/202068) and CNPq (307044/2015-7 and 303646/2019-5).

\section{Availability of data and materials}

All data generated and/or analyzed during this study are included in this published article.

\section{Ethics approval and consent to participate}

All the animal procedures were performed in full compliance with the institutional guidelines and were approved by the Institutional Animal Care and Use Committee (number: 8866221018).

\section{Consent for publication}

Not applicable

\section{Competing interests}

The authors declare that they have no competing interests.

\section{Author details}

${ }^{1}$ Interdisciplinary Center for Gene Therapy, Escola Paulista de Medicina, Universidade Federal de São Paulo, São Paulo, Brazil. ${ }^{2}$ Department of Physiological Sciences, Faculdade de Ciências Medicas da Santa Casa de São Paulo, São Paulo, Brazil. ${ }^{3}$ Department of Microbiology, Immunology and Parasitology, Escola Paulista de Medicina, Universidade Federal de Sao Paulo, São Paulo, Brazil. ${ }^{4}$ Department of Kinesiology and Nutrition, University of Illinois at Chicago, Chicago, USA. ${ }^{5}$ Department of Biophysics, Escola Paulista de Medicina, Universidade Federal de São Paulo, Rua Mirassol 207, São Paulo, SP 04044-010, Brazil.

Received: 28 July 2020 Accepted: 22 October 2020

Published online: 06 November 2020

\section{References}

1. Yelin $E$, Herrndorf A, Trupin L, et al. A national study of medical care expenditures for musculoskeletal conditions: the impact of health insurance and managed care. Arthritis Rheum. 2001;44:1160-9.

2. Jarvinen TA, Jarvinen $T L$, Kaariainen $M$, et al. Muscle injuries: biology and treatment. Am J Sports Med. 2005;33:745-64.

3. Jackson DW, Feagin JA. Quadriceps contusions in young athletes. Relation of severity of injury to treatment and prognosis. J Bone Joint Surg Am. 1973;55:95-105.

4. Jarvinen MJ, Lehto MU. The effects of early mobilisation and immobilisation on the healing process following muscle injuries. Sports Med. 1993;15:78-89.

5. Nozaki M, Li Y, Zhu J, et al. Improved muscle healing after contusion injury by the inhibitory effect of suramin on myostatin, a negative regulator of muscle growth. Am J Sports Med. 2008;36:2354-62.

6. Kasemkijwattana C, Menetrey J, Somogyl G, et al. Development of approaches to improve the healing following muscle contusion. Cell Transplant. 1998;7:585-98.

7. Lawrence T, Natoli G. Transcriptional regulation of macrophage polarization: enabling diversity with identity. Nat Rev Immunol. 2011;11:750-61.

8. Mantovani A, Sica A, Sozzani S, et al. The chemokine system in diverse forms of macrophage activation and polarization. Trends Immunol. 2004;25: 677-86

9. Wynn TA, Vannella KM. Macrophages in tissue repair, regeneration, and fibrosis. Immunity. 2016:44:450-62.

10. Arnold L, Henry A, Poron F, et al. Inflammatory monocytes recruited after skeletal muscle injury switch into antiinflammatory macrophages to support myogenesis. J Exp Med. 2007;204:1057-69.

11. Novak ML, Weinheimer-Haus EM, Koh TJ. Macrophage activation and skeletal muscle healing following traumatic injury. J Pathol. 2014;232:344-55.

12. Chazaud B, Sonnet C, Lafuste $P$, et al. Satellite cells attract monocytes and use macrophages as a support to escape apoptosis and enhance muscle growth. J Cell Biol. 2003;163:1133-43.

13. Song $E$, Ouyang $N$, Horbelt $M$, et al. Influence of alternatively and classically activated macrophages on fibrogenic activities of human fibroblasts. Cell Immunol. 2000;204:19-28.

14. Cantini M, Giurisato E, Radu C, et al. Macrophage-secreted myogenic factors: a promising tool for greatly enhancing the proliferative capacity of myoblasts in vitro and in vivo. Neurol Sci. 2002;23:189-94.

15. Verreck FA, de Boer T, Langenberg DM, et al. Human IL-23-producing type 1 macrophages promote but IL-10-producing type 2 macrophages subvert immunity to (myco)bacteria. Proc Natl Acad Sci U S A. 2004;101:4560-5.

16. Krausgruber T, Blazek K, Smallie T, et al. IRF5 promotes inflammatory macrophage polarization and TH1-TH17 responses. Nat Immunol. 2011;12: 231-8. 
17. Sacramento CB, Cantagalli VD, Grings M, et al. Granulocyte-macrophage colony-stimulating factor gene based therapy for acute limb ischemia in a mouse model. J Gene Med. 2009;11:345-53.

18. Shen W, Li Y, Tang Y, et al. NS-398, a cyclooxygenase-2-specific inhibitor, delays skeletal muscle healing by decreasing regeneration and promoting fibrosis. Am J Pathol. 2005;167:1105-17.

19. Yasumura EG, Stilhano RS, Samoto $W$, et al. Treatment of mouse limb ischemia with an integrative hypoxia-responsive vector expressing the vascular endothelial growth factor gene. PLoS One. 2012;7:e33944.

20. Sacramento CB, da Silva FH, Nardi NB, et al. Synergistic effect of vascular endothelial growth factor and granulocyte colony-stimulating factor double gene therapy in mouse limb ischemia. J Gene Med. 2010;12:310-9.

21. Martins L, Martin PK, Han SW. Angiogenic properties of mesenchymal stem cells in a mouse model of limb ischemia. Methods Mol Biol. 2014;1213:147-69.

22. Panduro $\mathrm{M}$, Benoist $\mathrm{C}$, Mathis $\mathrm{D}$. Treg cells limit IFN-gamma production to control macrophage accrual and phenotype during skeletal muscle regeneration. Proc Natl Acad Sci U S A. 2018;115:E2585-93.

23. Roszer $\mathrm{T}$. Understanding the mysterious $\mathrm{M} 2$ macrophage through activation markers and effector mechanisms. Mediat Inflamm. 2015;2015:816460,

24. Reidy PT, McKenzie Al, Mahmassani ZS, et al. Aging impairs mouse skeletal muscle macrophage polarization and muscle-specific abundance during recovery from disuse. Am J Physiol Endocrinol Metab. 2019;317:E85-98.

25. Tidball JG, Villalta SA. Regulatory interactions between muscle and the immune system during muscle regeneration. Am J Physiol Regul Integr Comp Physiol. 2010;298:R1173-87.

26. Olfert IM, Howlett RA, Tang K, et al. Muscle-specific VEGF deficiency greatly reduces exercise endurance in mice. J Physiol. 2009:587:1755-67.

27. Lygate CA, Aksentijevic D, Dawson D, et al. Living without creatine: unchanged exercise capacity and response to chronic myocardial infarction in creatine-deficient mice. Circ Res. 2013;112:945-55.

28. Manalo DJ, Rowan A, Lavoie T, et al. Transcriptional regulation of vascular endothelial cell responses to hypoxia by HIF-1. Blood. 2005;105:659-69.

29. Banquet $S$, Gomez E, Nicol L, et al. Arteriogenic therapy by intramyocardia sustained delivery of a novel growth factor combination prevents chronic heart failure. Circulation. 2011;124:1059-69.

30. Peng B, Zhao Y, Lu H, et al. In vivo plasmid DNA electroporation resulted in transfection of satellite cells and lasting transgene expression in regenerated muscle fibers. Biochem Biophys Res Commun. 2005;338:1490-8.

31. Takahashi T, Kalka C, Masuda H, et al. Ischemia- and cytokine-induced mobilization of bone marrow-derived endothelial progenitor cells for neovascularization. Nat Med. 1999;5:434-8.

32. McCourt M, Wang JH, Sookhai S, et al. Activated human neutrophils release hepatocyte growth factor/scatter factor. Eur J Surg Oncol. 2001;27:396-403.

33. Hoefer IE, Grundmann S, van Royen N, et al. Leukocyte subpopulations and arteriogenesis: specific role of monocytes, lymphocytes and granulocytes. Atherosclerosis. 2005;181:285-93.

34. Bergmann $\mathrm{CE}$, Hoefer IE, Meder B, et al. Arteriogenesis depends on circulating monocytes and macrophage accumulation and is severely depressed in op/op mice. J Leukoc Biol. 2006:80:59-65.

35. Miller KJ, Thaloor D, Matteson S, et al. Hepatocyte growth factor affects satellite cell activation and differentiation in regenerating skeletal muscle. Am J Physiol Cell Physiol. 2000;278:C174-81.

36. Zhan $Y, X u Y$, Lew $A M$. The regulation of the development and function of dendritic cell subsets by GM-CSF: more than a hematopoietic growth factor. Mol Immunol. 2012:52:30-7.

37. Hong IS. Stimulatory versus suppressive effects of GM-CSF on tumor progression in multiple cancer types. Exp Mol Med. 2016:48:e242.

38. Hercus TR, Thomas D, Guthridge MA, et al. The granulocyte-macrophage colony-stimulating factor receptor: linking its structure to cell signaling and its role in disease. Blood. 2009;114:1289-98.

39. Bhattacharya P, Budnick I, Singh M, et al. Dual role of GM-CSF as a proinflammatory and a regulatory cytokine: implications for immune therapy. J Interf Cytokine Res. 2015;35:585-99.

40. Mellman I, Steinman RM. Dendritic cells: specialized and regulated antigen processing machines. Cell. 2001;106:255-8

41. Walker F, Burgess AW. Internalisation and recycling of the granulocytemacrophage colony-stimulating factor (GM-CSF) receptor on a murine myelomonocytic leukemia. J Cell Physiol. 1987;130:255-61.

42. Guthridge MA, Powell JA, Barry EF, et al. Growth factor pleiotropy is controlled by a receptor Tyr/Ser motif that acts as a binary switch. EMBO J. 2006;25:479-89.
43. Brigitte $M$, Schilte $C$, Plonquet $A$, et al. Muscle resident macrophages control the immune cell reaction in a mouse model of notexin-induced myoinjury. Arthritis Rheum. 2010;62:268-79.

44. Honda H, Kimura H, Rostami A. Demonstration and phenotypic characterization of resident macrophages in rat skeletal muscle. Immunology. 1990;70:272-7.

45. McLennan IS. Resident macrophages (ED2- and ED3-positive) do not phagocytose degenerating rat skeletal muscle fibres. Cell Tissue Res. 1993; 272:193-6.

46. Pimorady-Esfahani A, Grounds MD, McMenamin PG. Macrophages and dendritic cells in normal and regenerating murine skeletal muscle. Muscle Nerve. 1997;20:158-66.

47. Saclier M, Cuvellier S, Magnan M, et al. Monocyte/macrophage interactions with myogenic precursor cells during skeletal muscle regeneration. FEBS J. 2013:280:4118-30.

48. Mills CD. Anatomy of a discovery: $\mathrm{m} 1$ and $\mathrm{m} 2$ macrophages. Front Immunol. 2015:6:212

49. Gonzalez A, Schelbert EB, Diez J, et al. Myocardial interstitial fibrosis in heart failure: biological and translational perspectives. J Am Coll Cardiol. 2018;71: 1696-706.

50. Liu Y. Renal fibrosis: new insights into the pathogenesis and therapeutics. Kidney Int. 2006;69:213-7.

51. Mauviel A. Transforming growth factor-beta: a key mediator of fibrosis. Methods Mol Med. 2005:117:69-80.

52. McLennan IS, Koishi K. The transforming growth factor-betas: multifaceted regulators of the development and maintenance of skeletal muscles, motoneurons and Schwann cells. Int J Dev Biol. 2002;46:559-67.

53. Li MO, Wan YY, Sanjabi S, et al. Transforming growth factor-beta regulation of immune responses. Annu Rev Immunol. 2006;24:99-146.

54. Rodgers BD, Wiedeback BD, Hoversten KE, et al. Myostatin stimulates, not inihibits, C2C12 myoblast proliferation. Endocrinology. 2014;155:670-5.

55. Schabort EJ, van der Merwe M, Loos B, et al. TGF-beta's delay skeletal muscle progenitor cell differentiation in an isoform-independent manner. Exp Cell Res. 2009;315:373-84.

56. Chegini $\mathrm{N}$, Tang XM, Ma C. Regulation of transforming growth factor-beta1 expression by granulocyte macrophage-colony-stimulating factor in leiomyoma and myometrial smooth muscle cells. J Clin Endocrinol Metab. 1999:84:4138-43.

57. Ripley D, Tang XM, Ma C, et al. The expression and action of granulocyte macrophage-colony stimulating factor and its interaction with TGF-beta in endometrial carcinoma. Gynecol Oncol. 2001;81:301-9.

58. Annes JP, Munger JS, Rifkin DB. Making sense of latent TGFbeta activation. J Cell Sci. 2003;116:217-24

59. de Sousa AR, Penalva LO, Marcotte EM, et al. Global signatures of protein and mRNA expression levels. Mol BioSyst. 2009;5:1512-26.

60. Vogel C, Marcotte EM. Insights into the regulation of protein abundance from proteomic and transcriptomic analyses. Nat Rev Genet. 2012;13:227-32.

61. Penn JW, Grobbelaar AO, Rolfe KJ. The role of the TGF-beta family in wound healing, burns and scarring: a review. Int J Burns Trauma. 2012;2:18-28.

62. Ferguson MW, O'Kane S. Scar-free healing: from embryonic mechanisms to adult therapeutic intervention. Philos Trans R Soc Lond Ser B Biol Sci. 2004; 359:839-50.

63. Ferguson MW, Duncan J, Bond J, et al. Prophylactic administration of avotermin for improvement of skin scarring: three double-blind, placebocontrolled, phase 1/II studies. Lancet. 2009:373:1264-74.

64. Yoon MS. mTOR as a key regulator in maintaining skeletal muscle mass. Front Physiol. 2017:8:788

65. Brook MS, Wilkinson DJ, Phillips BE, et al. Skeletal muscle homeostasis and plasticity in youth and ageing: impact of nutrition and exercise. Acta Physiol (Oxf). 2016;216:15-41.

66. Richard-Bulteau H, Serrurier B, Crassous B, et al. Recovery of skeletal muscle mass after extensive injury: positive effects of increased contractile activity. Am J Physiol Cell Physiol. 2008;294:C467-76.

\section{Publisher's Note}

Springer Nature remains neutral with regard to jurisdictional claims in published maps and institutional affiliations. 